\title{
Aiding Off-Road Inertial Navigation with High Performance Models of Wheel Slip
}

\author{
Forrest Rogers-Marcovitz, Michael George, Neal Seegmiller, and Alonzo Kelly ${ }^{1}$
}

\begin{abstract}
When GPS, or other absolute positioning, is unavailable, terrain-relative velocity is crucial for dead reckoning and the vehicle's pose estimate. Unfortunately, the positiondenied accuracy of the inertial navigation system (INS) is governed by the performance of the linear velocity aiding sources, such as wheel odometry, which are typically corrupted by large systematic errors due to wheel slip. As a result, affordable terrestrial inertial navigation is ineffective in estimating position when denied position fixes for an extended period of time. For mobile robots, the mapping between inputs and resultant behavior depends critically on terrain conditions which vary significantly over time and space which cannot be pre-programmed. Past work has used Integrated Perturbative Dynamics (IPD) to identify successively systematic and stochastic models of wheel slip, but treated the pose filter only as input without improving the odometry measurements used for vehicle navigation. We present a unique approach of a predictive vehicle slip model in a delayed state extended Kalman filter. The relative pose difference between the current state and delayed state is used as a measurement update to the vehicle slip model. These results create an opportunity to compensate for wheel slip effects in terrestrial inertial navigation. This paper presents the design, calibration, and verification of such a system and concludes that the position-denied performance of the compensated system is far superior.
\end{abstract}

\section{INTRODUCTION}

For a ground mobile robot, inertial navigation can provide excellent high frequency attitude information necessary for processing perception data and vehicle stability, high frequency linear and angular velocity information for vehicle control, and a capacity to dead reckon both heading and position when GPS, or other position fixes, are unavailable.

Today's affordable gyro technology is impressive. Tactical grade MEMS gyros have bias stabilities under 10 degrees per hour while fiber optic gyros are an order of magnitude smaller. The impact of this on mobile robots is that even heading, the most difficult of the three orientation angles, can be well estimated, without any external fixes, for many minutes.

The same is not true of accelerometers. The process of integrating accelerometer indications twice with respect to time magnifies errors to such a degree that an IMU is a poor surrogate for GPS for more than a few seconds of time. Even with wheel slippage, the process of integrating accelerometers once with respect to time is a poor surrogate for wheel odometry. Indeed, a relatively high performance tactical grade IMU cannot compete with odometry for more than a few seconds, and moreover, when pose fixes from GPS

\footnotetext{
${ }^{1}$ All Authors are with the Robotics Institute, Carnegie Mellon University, Pittsburgh, PA, PA \{forrest, mgeorge, nseegmiller, alonzo\} $\mathrm{a}_{\mathrm{cmu}}$.edu
}

or other sources are absent, the dead reckoning performance of the entire system depends critically on the performance of odometry - as our results will show.

In the absence of position measurements ("fixes"), there are only two ways to get good position information - by integrating good velocity (or relative positions) once or by integrating very good acceleration twice. The second option requires navigation grade components that can cost twice as much as an automobile. The first option, for a ground robot, can be achieved with visual odometry [1], using cameras or lidar, under certain conditions, or with good wheel odometry. However, lidar odometry requires sufficient geometric texture everywhere, and camera odometry requires ambient light and sufficient features everywhere. Visual odometry is not always an option but can always complement wheel odometry. Wheel odometry does not perform well under conditions of slip. It has been shown that the dominant component of slip is often rotational for a skid-steered or tracked vehicle [2]. A gyro measures rotational velocity well; so sensor fusion is likely to be valuable even off-road. Furthermore, we have already argued that even uncompensated odometry outperforms free inertial derived translation.

For accurate results where the position does not drift quickly without position fixes, modeling wheel slip is an important part of pose estimation for outdoor mobile robots. We use Integrated Perturbative Dynamics (IPD) to learn the mapping between control inputs and resultant vehicle motion by calibrating the vehicle's mobility model online using an extended Kalman filter. Past work has used IPD to identify systematic and stochastic models of wheel slip [2], powertrain dynamics, and odometry parameters [3].

In past work on IPD, the vehicle pose system has been treated strictly as an input to the IPD filter, but the learned mobility models can improve the vehicle allied odometry by including predicted vehicle-terrain interactions. By combining pose estimation and model identification into a unified filter, we show that the measurement and system modeling uncertainty is correctly handled which leads to superior pose estimates from dead-reckoning.

\section{A. Vehicle System Modeling}

The aspects of wheel-terrain interaction that are needed for accurate ground robot models are neither well known nor easily measurable in realistic situations. Published methods are mostly concerned with robust path-following (e.g. [4]), or the estimation of instantaneous wheel slip for feedback controllers. Some have developed terramechanics-based models for slip estimation that require knowledge of tire constants 
and soil parameters [5]. Some methods lump all unknown tire and soil parameters into slip ratios and angles; extended Kalman filters have been developed for real-time estimation of slip ratios and angles using velocity measurements [6] [7]. Scheding et. al. [8] developed a slip model for Load, Haul, and Dump trucks (LHD) by estimating the current slip angles of front and rear axles separately; the slip angles were allowed to change over time by treating errors in slip as random walks.

Our approach relies on compensating a 3D wheel odometry solution for wheel slip. We deal with the case of arbitrary terrain and presently do not try to separate the effects of vehicle and terrain. Our slip models try to account for the effects of all of the forces on the vehicle, rather than just those directly related to the controls. Notably, the composite effects of gravity and lateral acceleration are modeled. We estimate instantaneous slip based on the inputs and IMUderived attitude, but the estimation algorithm is calibrated in an on-line process using a delayed state filter as described below.

The calibration of our slip model is based on the IPD approach described in [2]. This approach uses an integral of the perturbative dynamics of $3 \mathrm{D}$ odometry in order to increase the sensitivity and conditioning of the mapping between the history of wheel slip along an arbitrary trajectory and the observed pose error that it causes a few seconds later. This method captures the underlying disturbance dynamics as a function of all of input space and is calibrated online based on trajectories executed under normal operation. In the past, the pose filter was separate from the vehicle system identification and treated only as a measurement input.

\section{B. Delayed State Filter}

Whenever a filter is provided with a sequence of measurements which is itself based on dead reckoning, the inevitable correlation of the measurement sequence will have to be treated correctly. One technique for doing so is the use of delayed states. This technique allows the filter to correctly extract the information about relative motion from the measurement without burdening the measurement system with the problem of computing its correlations.

We use a delayed state EKF formulation that has been referred to as stochastic cloning in [12] and [13]. Helmick et. al. [14] have also used a delayed state formulation for slip-compensated path following on planetary exploration rovers by calculating a slip vector via differencing the current Kalman filter estimate from the kinematic estimate. This method, along with most classical model identification techniques, estimates the current slip vector, or combination of slip angle and slip ratio, by using the differential equation directly, which requires observations of $\underline{x}$ and $\underline{\dot{x}}$. Often $\underline{\dot{x}}$ can not be measured directly, so measurements of $\underline{x}$ are numerically differentiated with respect to time.

Instead of using the delayed state to add a visual odometry measurement update, we will use the relative pose difference between the current state and delayed state as a measurement update to the slip model. The measurement update can occur at a fixed time interval or after a given distance has been traveled. Since we use RTK GPS measurements in this paper, the delayed state formulation is less important, due to weaker correlations, but this arrangement is the most general and allows for additional sensing with few changes. It should be noted that if visual odometry was used as an input to the pose system, such a measurement could be used to update the pose and slip model simultaneously. The delayed state formulation enables future investigations into calibrating slip using visual odometry, less accurate GPS and perhaps even just the integrated inertial navigation system.

In the following sections, we present an approach to unified pose estimation and vehicle model identification. Section II introduces the vehicle system model along with the corresponding linearized error dynamics and perturbation model parameterization. Section III presents the delayed state Kalman filter which both estimates the vehicle's pose and identifies the vehicle's perturbation model. Experimental results are presented in Section IV, followed by the conclusion and discussion of future work.

\section{Vehicle System Modeling}

For a vehicle moving in contact with a surface, there are three degrees of freedom of motion as long as the vehicle remains in contact with the local tangent plane.

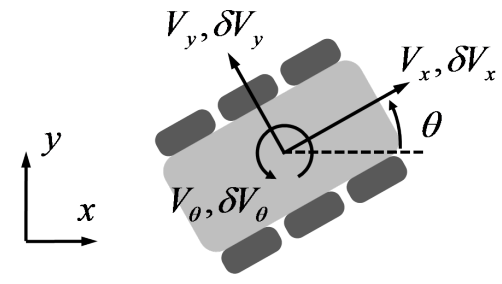

Fig. 1. Vehicle inputs and perturbations. Three degrees of freedom remain in the general case after terrain contact is enforced. Velocity inputs and disturbances are expressed in the body frame.

It is most natural to express actuation in the body frame. Given the vehicle's commanded linear and angular velocities, we have the following unconstrained kinematic differential equation for the time derivatives of $2 \mathrm{D}$ position and heading with respect to a ground-fixed reference frame:

$$
\underline{\dot{\rho}}=B(\gamma, \beta, \theta) \cdot \underline{u}
$$

or, expressed in full:

$$
\begin{aligned}
{\left[\begin{array}{l}
\dot{x} \\
\dot{y} \\
\dot{\theta}
\end{array}\right]=\left[\begin{array}{ccc}
c \theta c \beta & c \theta s \beta s \gamma-s \theta c \gamma & 0 \\
s \theta c \beta & s \theta s \beta s \gamma+c \theta c \gamma & 0 \\
0 & 0 & \frac{c \gamma}{c \beta}
\end{array}\right]\left[\begin{array}{l}
V_{x} \\
V_{y} \\
V_{\theta}
\end{array}\right] } \\
c=\cos (), \quad s=\sin (), \quad \gamma=\text { roll, } \beta=\text { pitch, } \theta=\text { heading }
\end{aligned}
$$

where $V_{x}$ is the forward velocity, $V_{y}$ is the lateral velocity in the body-left direction, and $V_{\theta}$ is the heading rate with positive rotation in the counter-clockwise direction - see Figure 1 . We will occasionally refer to this model in the sequel as 3D odometry. This system is nonlinear because the heading angle appears in the coefficient matrix. It should 
be noted that we chose a velocity-driven model instead of a comprehensive physics-based model for simplicity and vehicle generality.

Since we are using the wheel odometry directly, the transformation from left and right wheel velocities to forward and angular velocity for a tracked vehicle will be needed according to:

$$
\left[\begin{array}{l}
V_{x} \\
V_{\theta}
\end{array}\right]=\left[\begin{array}{cc}
\frac{1}{2} & \frac{1}{2} \\
-\frac{1}{W} & \frac{1}{W}
\end{array}\right]\left[\begin{array}{l}
V_{l} \\
V_{r}
\end{array}\right]
$$

where $V_{l}$ and $V_{r}$ are the left and right encoder speeds, and the wheel base, $W$, is the distance between the centers of the two tracks.

\section{A. Linearized Error Dynamics}

We will briefly cover the mathematics of linearizing the error dynamics of the systematic vehicle model - see [2] for a full development and explanation of the equations. Pose error of a path segment is attributed to the initial pose measurement error and input velocity perturbations, $\delta u$. Including these velocity perturbations, the kinematic differential equation becomes:

$$
\underline{\dot{\rho}}=B(\gamma, \beta, \theta) \cdot(\underline{u}+\delta \underline{u}), \quad \delta \underline{u}=\left[\begin{array}{l}
\delta V_{x} \\
\delta V_{y} \\
\delta V_{\theta}
\end{array}\right]
$$

In general form, the kinematic differential equation (2) can be written:

$$
\underline{\dot{\rho}}=\underline{f}(\underline{\rho}(t), \underline{u}(t))
$$

Recall that the state $(\rho)$ is the pose of the vehicle in the ground-fixed reference frame and the inputs $(\underline{u})$ are the linear and angular velocities in the body frame. By differentiating (5), we obtain the linearized error dynamics for deterministic or systematic error:

$$
\delta \underline{\dot{\rho}}=F(t) \delta \underline{\rho}(t)+G(t) \delta \underline{u}(t)
$$

where $F$ and $G$ are Jacobian matrices:

$$
\begin{aligned}
& F=\frac{\partial \underline{f}}{\partial \underline{\rho}}=\left[\begin{array}{ccc}
0 & 0 & -\dot{y} \\
0 & 0 & \dot{x} \\
0 & 0 & 0
\end{array}\right] \\
& G=\frac{\partial \underline{f}}{\partial \underline{u}}=B(\gamma, \beta, \theta)
\end{aligned}
$$

This allows us to define the transition matrix, $\Phi$, and the input transition matrix, $\Gamma$ :

$$
\begin{gathered}
\Phi(t, \tau)=e^{\int_{\tau}^{t} F(\zeta) d \zeta}=\left[\begin{array}{ccc}
1 & 0 & -(y(t)-y(\tau)) \\
0 & 1 & (x(t)-x(\tau)) \\
0 & 0 & 1
\end{array}\right] \\
\Gamma(t, \tau)=\Phi(t, \tau) B(\gamma, \beta, \theta)
\end{gathered}
$$

Using the transition matrices, the solution to the first-order differential equation (6) is the following vector convolution integral:

$$
\delta \underline{\rho}(t)=\Phi\left(t, t_{0}\right) \delta \underline{\rho}\left(t_{0}\right)+\int_{t_{0}}^{t} \Gamma(t, \tau) \delta \underline{u}(\tau) d \tau
$$

\section{B. Parameterization}

Of course, wheel slip is not constant but depends on the commanded trajectory and local terrain physical properties. Accordingly, we parameterize the systematic component of $\delta \underline{u}$ over velocities and accelerations predicted from the encoder measurements along with components of the gravity vector, which are available from the pose filter:

$$
\begin{aligned}
& \delta \underline{u}=\left[\begin{array}{c}
V_{s, x} \\
V_{s, y} \\
V_{s, \theta}
\end{array}\right]=C \underline{\alpha} \\
& C=\left[\begin{array}{lll}
\underline{c}_{x} & & \\
& \underline{c}_{y} & \\
& & \underline{c}_{\theta}
\end{array}\right] \\
& \underline{c}_{x}=\left[\begin{array}{llll}
V_{x} & \left|V_{\theta}\right| & \left(V_{x}\left|V_{\theta}\right|\right) & g_{x}
\end{array}\right] \\
& \underline{c}_{y}=\left[\begin{array}{llll}
V_{x} & V_{\theta} & \left(V_{x} V_{\theta}\right) & g_{y}
\end{array}\right] \\
& \underline{c}_{\theta}=\left[\begin{array}{lllll}
V_{x} & V_{\theta} & \left(V_{x} V_{\theta}\right) & g_{x} & g_{y}
\end{array}\right]
\end{aligned}
$$

$C$ is a $3 \times 13$ matrix in which all off-diagonal element blocks are zero. The three slip velocities are treated independent of each other and are parameterized by the row vectors $\underline{c}_{x}, \underline{c}_{y}$, and $\underline{c}_{\theta}$. The slip rate parameter vector, $\underline{\alpha}$, includes the coefficients that are learned by the online filter.

This parameterization works well in practice but also makes intuitive sense. Wheel slip is fundamentally caused by forces acting on the vehicle. The velocity terms $V_{x}$ and $V_{\theta}$ are included because frictional contact forces are proportional to them; these terms also capture errors in the odometry model such as mis-calibrated wheel radius or rate scaling factors. The use of absolute values in the parameterization of forward slip makes it not dependent on the direction of turn. Centripetal acceleration $\left(V_{x} V_{\theta}\right)$ and the components of the gravity vector $\left(g_{x}, g_{y}\right)$ captures the main applied noncontact forces.

\section{Delayed State Kalman Filter}

\section{A. Inertial Prediction}

The system state for pose estimation, $\underline{x}$, is defined by the current orientation, position and velocity of the IMU in the vehicle frame along with the slip rate parameters defined above:

$$
\underline{x}=\left[\begin{array}{llll}
\underline{\Psi} & \underline{R}^{n} & \underline{V}^{n} & \underline{\alpha}
\end{array}\right]
$$

where $\underline{\Psi}=[\gamma, \beta, \theta]$ are the roll, pitch, and heading Euler angles relating the navigation frame (n) to the vehicle fixed body frame (b). $\underline{R}^{n}$ is the position in the navigation frame, and $\underline{V}^{n}$ is linear velocities in the navigation frame.

While it is typical in inertial navigation, we do not include the inertial sensor biases in the state vector. The reason for this is that our gyros are so good that vehicle heading error can be maintained within a few degrees for almost an hour. Unless we intended to operate fix denied for longer than this, there is little value in estimating gyro biases. Accelerometer biases are omitted for the opposite reason. These sensors are so poorly equipped to estimate position, even when 
calibrated in-run, that the odometry aided system ignores the accelerometers anyway. This is particularly true when odometry is well calibrated. It is straight forward to add the biases to the filter if desired.

State derivatives, which define the kinematic relations with regard to the current state and IMU measurements, are defined by:

$$
\underline{\dot{x}}=\left[\begin{array}{c}
\mathrm{E}\left(\underline{\omega}^{b}-\underline{\Omega}^{b}+\underline{n}_{\underline{\omega}^{b}}\right) \\
C_{b}^{n}\left(\underline{f}^{b}+\underline{n}_{f^{b}}\right)-2 \underline{\Omega}^{n} \times \underline{V}^{n}+\underline{g}^{n} \\
\underline{n} \underline{\alpha}
\end{array}\right]
$$

where $f^{b}, \underline{\omega}^{b}$ are the accelerometer and gyroscope measurements respectively, $E$ is a matrix that relates Euler angle rates to gyroscope measured rotation rates, $C_{b}^{n}$ is a rotation matrix, formed from the Euler angles, that relates the IMUvehicle frame to the navigation frame, $g^{n}$ is the known local gravity vector (incorporating centripetal acceleration terms) and $\underline{\Omega}$ is the known earth-rate vector (See [15] for details). Gravity and earth rotation rate in the navigation frame are provided as deterministic inputs. The slip rate parameters only change when new measurements arrive, but their uncertainty is increased as they are treated as random walk variables.

Uncertainty is incorporated with Gaussian additive noises, $\underline{n}_{*}$ on $\underline{\omega}^{b}$ and $f^{b}$ which represent true noise in the sensor. Uncertainty is represented by the covariance of the states

$$
P=E\left[(\underline{x}-\underline{\bar{x}})(\underline{x}-\underline{\bar{x}})^{\top}\right]
$$

where $E$ in the last expression represents the expected value operator, not to be confused with the Euler angle rate matrix $\mathrm{E}$ presented earlier. The covariance is propagated by numerical integration of the Lyapunov equation

$$
\dot{P}=F P+P F^{\top}+G Q G^{\top}
$$

where the following definitions apply:

$$
\begin{gathered}
F=\left[\begin{array}{cccc}
\frac{\partial\left(\mathrm{E}\left(\underline{\omega}^{b}-\underline{\Omega}^{b}\right)\right)}{\partial \underline{\Psi}} & 0 & 0 & 0 \\
0 & 0 & I & 0 \\
\frac{\partial\left(C_{b}^{n}\left(f^{b}\right)\right)}{\partial \underline{\Psi}} & 0 & {\left[-2 \underline{\Omega}^{n} \times\right]} & 0 \\
0 & 0 & 0 & 0
\end{array}\right] \\
G=\left[\begin{array}{ccc}
E & 0 & 0 \\
0 & 0 & 0 \\
0 & C_{b}^{n} & 0 \\
0 & 0 & I
\end{array}\right]
\end{gathered}
$$

These are simply the Jacobians of the state derivative relative to state and noise respectively. Finally

$$
Q=\operatorname{diag}\left(E\left[\underline{n}_{\underline{\omega}^{b}} \underline{n}_{\underline{\omega}^{b}}^{\top}\right], E\left[\underline{n}_{f^{b}} \underline{n}_{\underline{f}^{b}}^{\top}\right], E\left[\underline{n}_{\underline{\alpha}} \underline{n}_{\underline{\alpha}}^{\top}\right]\right) .
$$

Where $\underline{n}_{\alpha}$ is the expected variance of how the slip parameters vary over time; it can be increased when it is known that the vehicle has driven from one terrain type to another. For clarity, the time dependence of these parameters is not explicit in the notation but it is seen that all the matrices contain time varying values.

\section{B. Delayed State Filter}

At initialization, the orientation and position states of the filter are copied such that a new state, $\underline{\hat{x}} \in \mathbb{R}^{28 \times 1}$, is created by appending some delayed states $\underline{x}^{d}$ to the original states

$$
\underline{\hat{x}}=\left[\begin{array}{llllll}
\underline{\Psi} & \underline{R}^{n} & \underline{V}^{n} & \underline{\alpha} & \underline{\Psi}^{d} & \underline{R}^{n, d}
\end{array}\right]^{\top}
$$

$\underline{x}^{d}=\left[\underline{\Psi}^{d}, \underline{R}^{n, d}\right]$ represents the delayed state from the beginning of the path segment used for model identification. Along the path segment, a history of roll and pitch angles, between the delayed and current states, is stored separately from the filter; these angles can come from either past IMU measurements or from placing the vehicle onto a elevation map. The state covariance is similarly obtained

$$
\hat{P}=T P T^{\top}=\left[\begin{array}{cc}
P^{o o} & P^{o d} \\
P^{o d^{\top}} & P^{d d}
\end{array}\right]
$$

where, we refer to states from the original state vector with superscript $o$ and delayed states with superscript $d$. Here

$$
T=\left[\begin{array}{cccc}
\ddots & & & \\
& I & & \\
& & \ddots & \\
I & 0 & 0 & 0 \\
0 & I & 0 & 0
\end{array}\right]
$$

is a $28 \times 22$ matrix designed to select the appropriate rows of orientation and position covariance for copying. The result is an identical duplication of position and orientation estimates with their associated variances and covariances at that instant in time. The original states evolve with new measurements, while the delayed states remain fixed, in effect saving the information available at the instant they were appended. That is

$$
\underline{\hat{x}}=\left[\begin{array}{c}
\mathrm{E}\left(\underline{\omega}^{b}-\underline{\Omega}^{b}+\underline{n}_{\underline{\omega}^{b}}\right) \\
\underline{V}_{b}^{n}\left(\underline{f}^{b}+\underline{n}_{f^{b}}\right)-2 \underline{\Omega}^{n} \times \underline{V}^{n}+\underline{g}^{n} \\
\underline{\underline{n}} \underline{\underline{0}} \\
\underline{0}
\end{array}\right] .
$$

The variances of the appended states are fixed (block diagonal elements) representing the uncertainty in the states at the instant they were appended. However the covariances (block off-diagonal) with the current state propagate as the current state continues to evolve

$$
\dot{\hat{P}}=\left[\begin{array}{cc}
F P^{o o}+P^{o o} F^{\top}+G Q G^{\top} & F P^{o d} \\
\left(F P^{o d}\right)^{\top} & 0
\end{array}\right]
$$

where the previous definitions of $F, G$ and $Q$ still apply. See [12] for additional details.

\section{Odometry Measurement}

Using the slip-compensated vehicle model, we can achieve improved dead-reckoning relative to naive integration of the odometry measurements (3). By assumption, the perturbative (i.e. slip) dynamics are determined by the odometry velocity and the roll and pitch measurements (12). This allows the 
filter to add the predicted perturbative velocities to the measurement velocity. The perturbed motion model includes odometry and the systematic velocity perturbations. In the navigation frame, this perturbed model is defined in (6).

We use the perturbed model for linear velocity measurements only, while relying on the more accurate gyroscope to indicate angular velocity.

$$
\begin{aligned}
C_{n}^{b} \underline{V}^{n} & =\left[\begin{array}{c}
V_{x}^{o} \\
0 \\
0
\end{array}\right]+\left[\begin{array}{c}
\delta V_{x} \\
\delta V_{y} \\
0
\end{array}\right] \\
h_{\text {odom }}(\underline{x}) & =\underline{z}_{\text {odom }}+\underline{n}_{\underline{V}^{o}}+\underline{n}_{\delta \underline{u}} \\
H_{\text {odom }} & =\left[\begin{array}{llllll}
\frac{\partial\left(C_{n}^{b} \underline{V}^{n}\right)}{\partial \underline{\Psi}} & \underline{0} & C_{n}^{b} & \underline{0} & \underline{0} & \underline{0}
\end{array}\right]
\end{aligned}
$$

The measurement uncertainty needs to take into account the uncertainty from odometry, $\underline{n}_{V^{o}}$, and the perturbative velocities given by the estimated coefficients, $\underline{n}_{\delta \underline{u}}=C P^{\alpha} C^{\top}$. $P^{\alpha}$ is the state uncertainty sub-matrix corresponding to the slip coefficients.

\section{Position Measurement}

We treat GPS measurements as standard position updates:

$$
\begin{aligned}
\underline{R}^{n} & =\underline{R}_{g p s}^{n} \\
h_{g p s}(\underline{x}) & =\underline{z}_{g p s}+\underline{n}_{R_{g p s}^{n}} \\
H_{g p s} & =\left[\begin{array}{llllll}
\underline{0} & \underline{I} & \underline{0} & \underline{0} & \underline{0} & \underline{0}
\end{array}\right]
\end{aligned}
$$

After the vehicle has traveled a sufficient distance, the state $\underline{\hat{x}}$ is given by

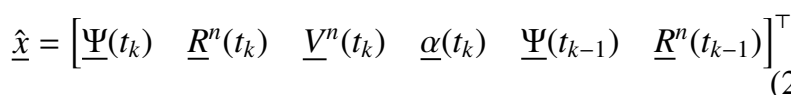

where $t_{k}$ refers the present state time and $t_{k-1}$ refers to the time of the delayed state.

At the end of each path segment, the difference between current state at the last key frame is used for slip model identification. To minimize uncertainty, the two key frames should occur right after GPS, or other position measurements are made. Starting at the last key frame, we propagate the pose forward using the past odometry measurements, along with measured roll and pitch history, and the current perturbation model coefficients.

$$
\underline{\rho}_{k, i p d}=\int_{t_{k-1}}^{t_{k}} B(\gamma(\tau), \beta(\tau), \theta(\tau)) \cdot(\underline{u}(\tau)+\delta \underline{u}(\tau, \alpha)) d \tau
$$

The Kalman filter measurement is the difference between final measured and final predicted poses $(x, y, \theta)$ :

$$
\begin{aligned}
\underline{z}_{i p d} & =\underline{\rho}_{k-1, i n s}^{-1} \otimes \underline{\rho}_{k, i n s} \\
h_{i p d}(\underline{x}) & =\underline{\rho}_{k, i p d}+\underline{n}_{i p d}
\end{aligned}
$$

Here, $\otimes$ refers to the pose addition found by multiplying the corresponding Homogeneous Transforms.

The measurement covariance is:

$$
\begin{aligned}
R_{i p d} & =E\left[\underline{n}_{i p d} \underline{n}_{i p d}^{\top}\right] \\
& =T_{i}^{n}\left(\hat{P}_{x, y, \theta}^{o o}+\Phi \hat{P}_{x, y, \theta}^{d d} \Phi^{\top}-\hat{P}_{x, y, \theta}^{o d} \Phi^{\top}-\Phi \hat{P}_{x, y, \theta}^{d o}\right)\left(T_{i}^{n}\right)^{\top}
\end{aligned}
$$

$$
T_{i}^{n}=\left[\begin{array}{ccc}
c\left(-\theta_{i}\right) & -s\left(-\theta_{i}\right) & 0 \\
s\left(-\theta_{i}\right) & c\left(-\theta_{i}\right) & 0 \\
0 & 0 & 1
\end{array}\right]
$$

where the four uncertainty terms, respectively, come from the current state, the delayed state, and the covariance between the two. The transition matrix, $\Phi$ (9), computes the effect of initial orientation uncertainty on final predicted state. $T_{i}^{n}$ transforms the uncertainty from the navigation frame to initial pose frame.

To obtain the measurement Jacobian $H$ we differentiate the predicted measurement with respect to the state $\underline{\alpha}$. We use Leibnitz's rule to move the derivative inside the integral.

$$
\begin{gathered}
J_{\alpha}=\frac{\partial \underline{h}(\underline{\alpha})}{\partial \underline{\alpha}}=\int_{t_{0}}^{t} \Gamma(t, \tau) \frac{\partial \underline{\underline{u}}(\underline{\alpha}, \tau)}{\partial \underline{\alpha}} d \tau=\int_{t_{0}}^{t} \Gamma(t, \tau) C(\tau) d \tau \\
H_{i p d}=\left[\begin{array}{llllll}
\underline{0} & \underline{0} & \underline{0} & J_{\alpha} & \underline{0} & \underline{0}
\end{array}\right]
\end{gathered}
$$

where $C(\tau)$ is defined by (13), and $\Gamma(t, \tau)$ is from (10).

\section{ExPERimental VAlidation}

Tracked vehicles are possibly the worst case for slip since they must slip by design during any turn. Data was collected on a custom surveillance robot similiar to the Foster-Miller TALON. Even though the IPD system identification algorithm was originally developed for skid-steered and Ackermann vehicles, the vehicle motion model is general enough to use directly on a tracked robot with no changes. The robot was instrumented with encoders on each track, GPS, and an Honeywell HG1930 IMU including accelerometers and gyros. The GPS data was post-processed with a nearby base station for high accuracy positioning. For purposes of this test, GPS was used in the filter only during the calibration phase. The robot was driven on soft dirt at relatively high commanded speeds of up to $1.7 \mathrm{~m} / \mathrm{s}$ and $3 \mathrm{rad} / \mathrm{s}$.

For the first four minutes after initialization, the complete filter was run using sensor inputs from the IMU, gyro, encoders and GPS; IPD calibrated the systematic slip model using the filter's delayed state at two second intervals. To track performance during calibration, after each IPD update, the vehicle's path was predicted 5 seconds into the future using odometry both with and without the calibrated vehicle model. As can be seen in the two scatter plots, Figures 2(a) and 2(b), the standard deviation of along track error and cross track error are reduced by $95 \%$ and $89 \%$, respectively, of the non-calibrated odometry error, using the current calibrated model. The standard deviation of heading prediction error is reduced by $70 \%$. The mean distance error is also reduced from $83 \mathrm{~cm}$ to $19 \mathrm{~cm}, 77 \%$, with a mean heading reduction of $83 \mathrm{mrad}$ to $16 \mathrm{mrad}, 81 \%$.

After the calibration phase, all GPS updates were disabled. To compare how well the calibrated vehicle model improved pose results, the pose-only filter was run in 5 different modes for the next minute: IMU Only, Odometry Only, Odometry+Slip, Odometry+IMU, Odometry+IMU+Slip. Slip refers to slip compensation based on the perturbative velocities estimated by the calibrated vehicle model. See Figures 3 


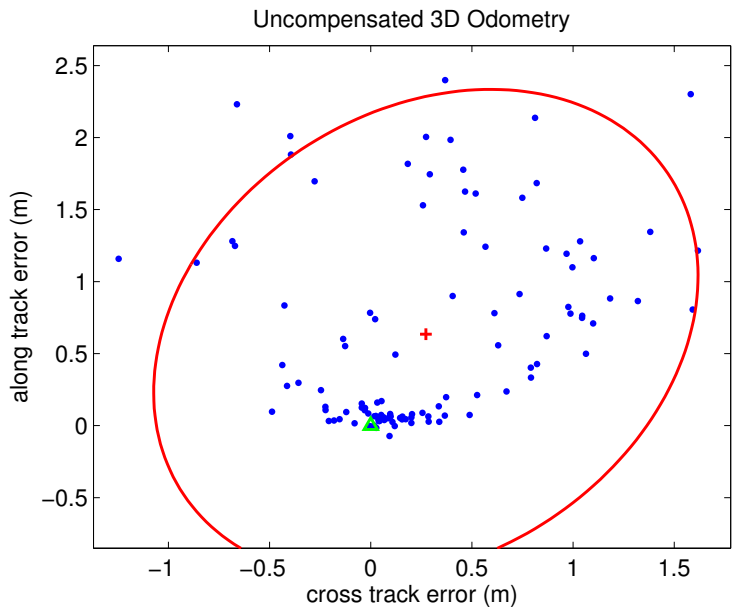

(a)

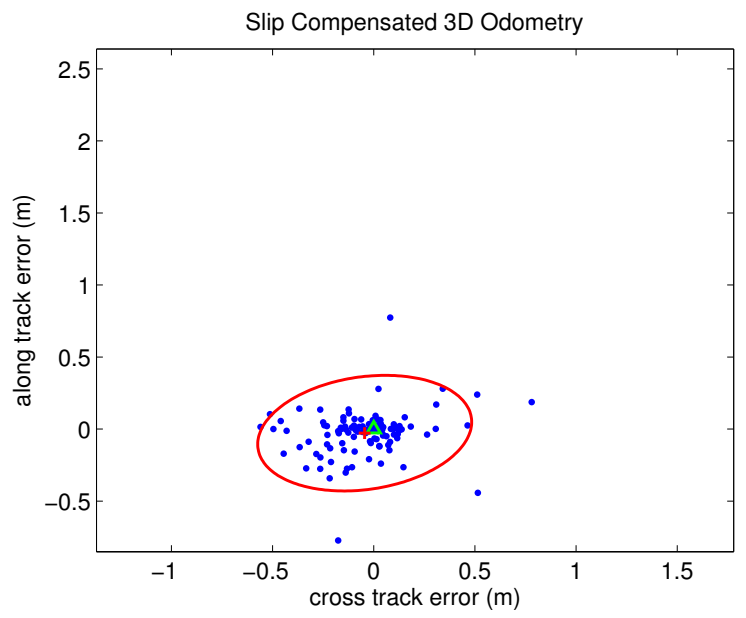

(b)

Fig. 2. Scatter plots of along track and cross track error for predicted pose error at the end of a five-second path segment. The two-standard deviation error ellipse of the points is shown by the solid red line. Figure (a) figure shows predicted pose using odometry only; figure (b) shows prediction error using odometry and the current estimate of the vehicle slip mode.

and 4 for results. Ground truth positioning provided by the differential GPS is shown by the thick blue line.

Table I presents the distance errors at the end of 60 seconds of the 5 different pose filters. The IMU Only solution quickly drifted away from the true solution and ended with an error of 48 meters. Free inertial navigation is not viable at all even with a relatively high performance IMU. The vehicle slip model produced the largest relative improvement when it aided the Odometry Only solution without the IMU, Figure 3. The Odometry Only solution overestimated rotations and suffered from frequent misalignments which caused an end error of $33.2 \%$ of distance traveled while the Odometry+Slip solution more closely tracked the true path and had a $5.7 \%$ distance traveled end error - a factor of 5.9 improvement.

The improvement produced by slip modeling when odometry and IMU sensors were included is only slight, from $0.31 \%$ before to $0.27 \%$ after, Figure 4 . The dominant component of slip is rotational and the gyro also measures this well,

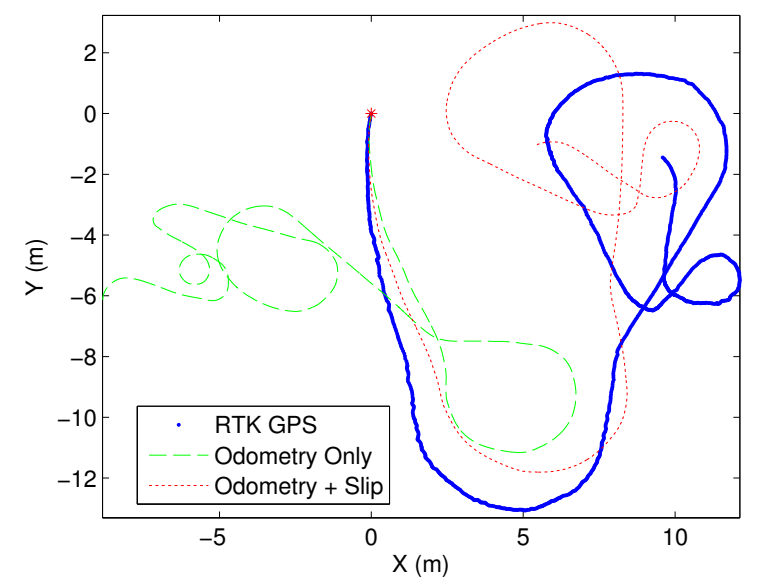

Fig. 3. Predicted path using vehicle odometry with and without the calibrated vehicle model.

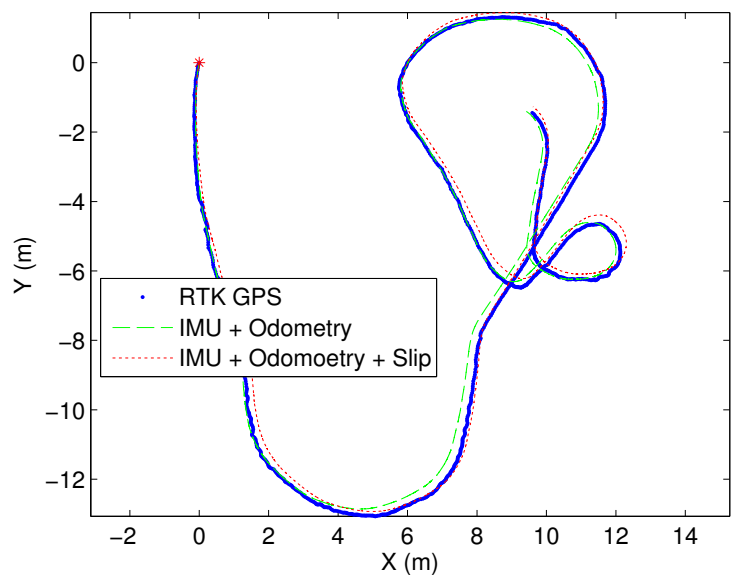

Fig. 4. Predicted path using IMU sensors and odometry with and without the calibrated vehicle model.

however the slip model still improves heading performance. This is because velocity is measured in the vehicle frame and transformed to the global frame in the IMU + Odometry filter measurements. This transformation is a function of heading, so even linear slip degrades IMU heading performance. A larger relative improvement would be expected with a lower performance IMU or a longer path.

To further compare the results of the IMU + Odometry solutions, the two solutions were propagated for 7 minutes. The absolute position error along the path is highly dependent of the path geometry. The remaining error is a semi-random process so there is always a chance that the errors in the uncompensated system will cancel and appear to work better. It is the average performance over a long period of time that matters. Figure 5 shows that the calibrated slip model solution had an position error less than the non-calibrated solution $90.3 \%$ of the time with a reduction in position error of $32 \%$ measured at every time-step along the path and final reduction of $76 \%$. The complete path is show in Figure 6. The end position error using the slip model was only $1.13 \%$ of distance traveled. 
TABLE I

End Path Error, Total Path Length: 57.35 meters, 60 seconds

\begin{tabular}{l||c|c}
\hline \hline Filter & Position Error $(\mathrm{m})$ & \% of distance traveled \\
\hline IMU Only & 48.23 & 84.09 \\
Odometry Only & 19.03 & 33.18 \\
Odometry+Slip & 3.25 & 5.67 \\
IMU+Odometry & 0.18 & 0.31 \\
IMU+Odometry+Slip & 0.15 & 0.27 \\
\hline
\end{tabular}

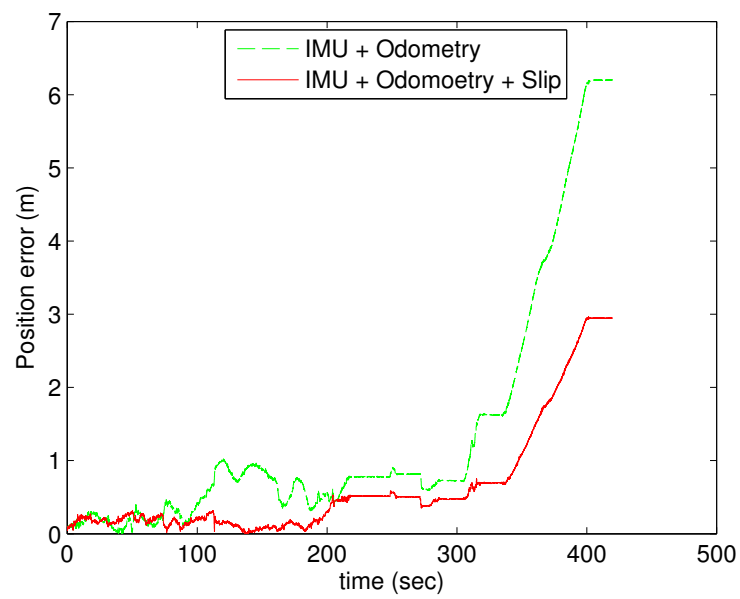

Fig. 5. Position Error along the path of the two IMU + Odometry solutions. The calibrated slip model had a position error less that the non-calibrated solution $90.3 \%$ of the time over the 313 meter path.

A case where we think slip modeling will make a large difference is combining it with visual odometry and wheel odometry. Visual odometry, which normally offers great short relative poses, often suffers from dropouts which can be detected. This allows the system to calibrate using good visual odometry relative poses and use the slip prediction model when frames are lost due to errors such as motion blur or bad lighting.

Thirty minutes of data was collected with the robot. During the thirty minutes, the robot's slip model was calibrated over four terrains: Dirt, Rock, Asphalt, and Grass. While driving on grass, the vehicle experienced slopes of up to 35 degrees. The complete results are not shown for brevity, but mobility calibration results over all four terrains are presented in [16]. Results from the four minute calibration phase and minute-long path were presented because they were the beginning of the data collection and also represented typical results seen across the dataset. It was shown that the mobility model parameters quickly converged as the robot drove between terrain types.

\section{Conclusion and Future Work}

In this paper we have shown that slip modeling can produce a significant improvement in odometry dead reckoning accuracy - even off road for a tracked vehicle with a high performance IMU. We have also argued that the fix-denied performance of aided terrestrial inertial navigation systems is governed in many cases by the performance of wheel odometry, and then showed that performance of inertial

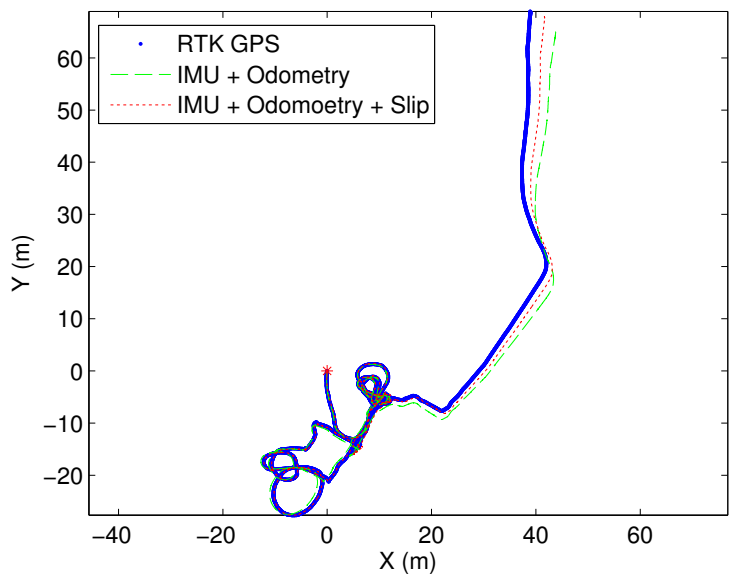

Fig. 6. Predicted 7 minute path using IMU sensors and odometry with and without the calibrated vehicle model.

navigation is also significantly improved with a calibrated model of slip.

We have shown that wheel slip model identification can be integrated successfully with pose in a single estimation system. We used RTK GPS measurements in this work, but it will also be interesting in future to see if lower quality measurements can calibrate models of slip well. There are two reasons to be optimistic. First, the capacity of the IPD approach to calibrate models of uncertainty suggests that the associated integrated filter can be well tuned. Second, the delayed state approach allows significant correlations to accumulate in order to disambiguate the likely causes of observed pose prediction error.

Our first goal was to demonstrate the viability and value of off-road slip modeling. Our IMU was one of the highest performance MEMS IMUs available on the market and slip modeling still mattered. Therefore, it seems that all land inertial navigation systems are likely to benefit from slip modeling. In addition, the improved models produced by this technique should lead to significant improvements in the performance of model predictive controllers, particularly in difficult terrains or during aggressive maneuvers.

In our experiments we used a clear calibration phase and then stopped calibration thereafter thus freezing the slip model. When the slip model is allowed to evolve indefinitely, interesting questions arise. First, can the slip models be calibrated continuously while the system operates - across transitions in terrain type. Second, how good do the pose sensors need to be to identify the slip model well. There is every reason to believe that when visual odometry can operate simultaneously with wheel odometry, calibration can proceed. Even more interesting is the question of whether good gyros alone will generate enough information to calibrate the slip models, because then the fusion of the INS and the slip models will be a self contained higher performance system requiring no new inputs except, possibly, the vehicle controls. 


\section{REFERENCES}

[1] J. Tardif, M. George, M. Laverne, A. Kelly, and A. Stentz, "A new approach to vision-aided inertial navigation," in International Conference on Intelligent Robots and Systems, 2010.

[2] N. Seegmiller, F. Rogers-Marcovitz, G. Miller, and A. Kelly, "A unified perturbative dynamics approach to vehicle model identification," in Proc. International Symposium on Robotics Research, Aug 2011.

[3] N. Seegmiller, F. Rogers-Marcovitz, and A. Kelly, "Online calibration of vehicle powertrain and pose estimation parameters using integrated perturbative dynamics," in Proc. International Conference on Robotics and Automation, May 2012.

[4] E. Lucet, C. Grand, D. Sallé, and P. Bidaud, "Dynamic sliding mode control of a four-wheel skid-steering vehicle in presence of sliding," in Proc. RoManSy, Tokyo, Japan, July 2008.

[5] G. Ishigami, A. Miwa, K. Nagatani, and K. Yoshida, "Terramechanicsbased model for steering maneuver of planetary exploration rovers on loose soil," J. Field Robotics, vol. 24, no. 3, pp. 233-250, 2007.

[6] J. Yi, H. Wang, J. Zhang, D. Song, S. Jayasuriya, and J. Liu, "Kinematic modeling and analysis of skid-steered mobile robots with applications to low-cost inertial-measurement-unit-based motion estimation," IEEE Transactions on Robotics, vol. 25, pp. 1087-1097, October 2009.

[7] C. B. Low and D. Wang, "Integrated estimation for wheeled mobile robot posture, velocities, and wheel skidding perturbations," in Proc. IEEE Intertnational Conference on Robotics and Automation, 2007, pp. 2355-2360.

[8] S. Scheding, G. Dissanayake, E. Nebot, and H. Durrant-Whyte, "Slip modelling and aided inertial navigation of an lhd," in Robotics and
Automation, 1997. Proceedings., 1997 IEEE International Conference on, vol. 3, apr 1997, pp. $1904-1909$ vol.3.

[9] L. Seneviratne, Y. Zweiri, S. Hutangkabodee, Z. Song, X. Song, S. Chhaniyara, S. Al-Milli, and K. Althoefer, "The modeling and estimation of driving forces for unmanned ground vehicles in outdoor terrain," International Journal of Modeling, Identification, and Control, vol. 6, no. 1, pp. 40-50, 2009.

[10] A. Angelova, L. Matthies, D. Helmick, G. Sibley, and P. Perona, "Learning to predict slip for ground robots," in Proc. IEEE International Conference on Robotics and Automation, 2006.

[11] M. Bode, "Learning the forward predictive model for an off-road skidsteer vehicle," Robotics Institute, Pittsburgh, PA, Tech. Rep. CMU-RITR-07-32, September 2007.

[12] A. I. Mourikis and S. I. Roumeliotis, "On the treatment of relative-pose measurements for mobile robot localization," in IEEE International Conference on Robotics and Automation, 2006, pp. 15-19.

[13] A. Mourikis, S. Roumeliotis, and J. Burdick, "Sc-kf mobile robot localization: A stochastic cloning kalman filter for processing relativestate measurements," Robotics, IEEE Transactions on, vol. 23, no. 4 , pp. $717-730$, aug. 2007.

[14] D. Helmick, S. Roumeliotis, Y. Cheng, D. Clouse, M. Bajracharya, and L. Matthies, "Slip-compensated path following for planetary exploration rovers," Advanced Robotics, vol. 20, no. 11, pp. 12571280, 2006.

[15] D. Titteron and J. Weston, Strapdown Inertial Navigation Technology, 2nd ed. Stevenage, UK: Institution of Electrical Engineers, 2004.

[16] F. Rogers-Marcovitz, N. Seegmiller, and A. Kelly, "Continuous vehicle slip model identification on changing terrains," in RSS 2012 Workshop on Long-term Operation of Autonomous Robotic Systems in Changing Environments, 2012. 